TRANSACTIONS OF THE

AMERICAN MATHEMATICAL SOCIETY

Volume 364, Number 11, November 2012, Pages 5995-6008

S 0002-9947(2012)05679-0

Article electronically published on June 12, 2012

\title{
DEGENERACY LOCI OF FAMILIES OF DIRAC OPERATORS
}

\author{
THOMAS G. LENESS
}

\begin{abstract}
Generalizing some results from R. Leung's thesis, we compute, in rational cohomology, the Poincaré dual of the degeneracy locus of the family of Dirac operators parameterized by the moduli space of projectively anti-selfdual $\mathrm{SO}(3)$ connections on a closed four-manifold. This should be a useful tool in comparing gauge theoretic invariants of smooth four-manifolds.
\end{abstract}

\section{INTRODUCTION}

The definition of the spin invariants of a smooth four-manifold, due to V. Pidstrigach and A. Tyurin in [29], is sufficiently similar to that of the Donaldson invariants to suggest that there must be a formula relating the two. In this note, we perform a computation which will be useful in deriving such an equality. This formula, together with relations between the Donaldson and Seiberg-Witten invariants and between the spin and Seiberg-Witten invariants given by the $\mathrm{SO}(3)$ monopole cobordism $([12,[11,8])$, should be an important tool for deriving more explicit forms of these relations and possibly constraints on these invariants.

For $E_{\kappa}^{w} \rightarrow X$ a complex, rank-two vector bundle over a smooth, closed, oriented, four-manifold, with $c_{1}\left(E_{\kappa}^{w}\right)=w$ and $\kappa=-\frac{1}{4}\left\langle p_{1}(\mathfrak{s u}(E)),[X]\right\rangle$, the Donaldson invariants $([3,21])$ are defined by integrating cohomology classes, the $\mu$-classes, over the moduli space $M_{\kappa}^{w}$ of projectively anti-self-dual connections on $E_{\kappa}^{w}$. If $(W, \rho)$ is a $\operatorname{spin}^{c}$ structure on $X$, then each unitary connection $A$ on $E_{\kappa}^{w}$ defines a Dirac operator on the $\operatorname{spin}^{u}$ structure $\mathfrak{t}=\left(W \otimes E, \rho \otimes \mathrm{id}_{E}\right)$ of index $n_{a}(\mathfrak{t})$. If $n_{a}(\mathfrak{t}) \leq 0$ and we denote $\Lambda=c_{1}\left(W^{+}\right)+c_{1}(E)$, then by [5, 31] the subspace $J_{\kappa}^{\Lambda, w} \subset M_{\kappa}^{w}$, defined in (2.3), of connections whose Dirac operator has one-dimensional kernel is, for generic perturbations, a smooth submanifold of codimension $2\left(1-n_{a}(\mathfrak{t})\right)$. Here, we generalize some results of [23] by computing the Poincaré dual of $J_{\kappa}^{\Lambda, w}$.

Theorem 1.1. Let $E_{\kappa}^{w} \rightarrow X$ be a complex, rank-two vector bundle over a smooth, closed, oriented, four-manifold with $b_{1}(X)=0$. Let $M_{\kappa}^{w}$ be the moduli space of projectively anti-self-dual connections on $E_{\kappa}^{w}$ and let $K_{\kappa}^{w} \subset M_{\kappa}^{w}$ be any compact, codimension-zero submanifold. Let $\mathfrak{t}$ be a spin ${ }^{u}$ structure on $X$ with $p_{1}(\mathfrak{t})=\kappa$, $c_{1}(\mathfrak{t})=\Lambda$, and $n_{a}(\mathfrak{t}) \leq 0$. Let $J_{\kappa}^{\Lambda, w} \subset M_{\kappa}^{w}$ be the degeneracy locus of the $\operatorname{spin}^{u}$ structure $\mathfrak{t}$. Then, as an element of rational cohomology, the Poincaré dual of $J_{\kappa}^{\Lambda, w}$ in $K_{\kappa}^{w}$ is

$$
(-1)^{1-n_{a}(\mathfrak{t})} \sum_{i+2 j+2 k=1-n_{a}(\mathfrak{t})} f_{i, 2 j, 2 k} \mu(\mathfrak{t})^{i} \smile \Omega^{j} \smile \mu(x)^{k},
$$

Received by the editors November 23, 2009 and, in revised form, March 4, 2011.

2010 Mathematics Subject Classification. Primary 53C07, 57R57, 58J05, 58J20, 58J52.

The author was supported in part by a Florida International University Faculty Research Grant and by NSF grant DMS \#0905786. 
where $\mu(\mathfrak{t})$ and $\Omega$ are the $\mu$-classes defined in (3.5) and the coefficients $f_{i, j, k}$ are determined by the recursion relation (3.13) and are given as

$$
F(x, y, z)=\sum_{i, j, k} f_{i, 2 j, 2 k} x^{i} y^{2 j} z^{2 k}=\exp \left(\frac{1}{2} x J_{1}(z)+\frac{1}{4} y^{2} J_{2}(z)+J_{3}(z)\right),
$$

where the functions $J_{i}(z)$ are given by

$$
\begin{aligned}
& J_{1}(z)=z^{-1} \tan ^{-1}(z), \\
& J_{2}(z)=z^{-3}\left(z-\tan ^{-1}(z)\right), \\
& J_{3}(z)=-\frac{1}{2} n_{a}(\mathfrak{t}) \ln \left(1+z^{2}\right)+\kappa\left(z^{-1} \tan ^{-1}(z)-1\right) .
\end{aligned}
$$

The spin invariants of [29] are defined by integrating $\mu$-classes, defined in (3.3), over the Uhlenbeck closure of $J_{\kappa}^{\Lambda, w}$ :

$$
P_{X}^{\Lambda, w}(z)=\left\langle\bar{\mu}(z),\left[\bar{J}_{\kappa}^{\Lambda, w}\right]\right\rangle .
$$

Theorem 1.1 implies that the Poincaré dual of $J_{\kappa}^{\Lambda, w}$ can be written as an expression in $\mu$-classes, which we denote as $\mu\left(T_{\kappa}^{\Lambda, w}\right)$. One might thus expect the Donaldson and spin invariants to be related by

$$
P_{X}^{\Lambda, w}(z)=\left\langle\bar{\mu}(z),\left[\bar{J}_{\kappa}^{\Lambda, w}\right]\right\rangle=\left\langle\bar{\mu}(z) \smile \bar{\mu}\left(T_{\kappa}^{\Lambda, w}\right),\left[\bar{M}_{\kappa}^{w}\right]\right\rangle=D_{X}^{w}\left(z T_{\kappa}^{\Lambda, w}\right) .
$$

However, R. Leung's thesis, [23, shows that (1.2) only holds when $n_{a}(\mathfrak{t})=0$. If $\mathcal{V}\left(T_{\kappa}^{\Lambda, w}\right)$ is the geometric representative of $\mu\left(T_{\kappa}^{\Lambda, w}\right)$ used to define the Donaldson invariant, then Theorem [1.1 shows that the intersections of $\mathcal{V}\left(T_{\kappa}^{\Lambda, w}\right)$ and $J_{\kappa}^{\Lambda, w}$ with any compact subset of $M_{\kappa}^{w}$ are cobordant, but the same is not true for their closures in the Uhlenbeck compactification. Thus, while Theorem 1.1 does not give a relation between the Donaldson and spin invariants, it does allow one to localize the error in (1.2) near the lower strata of the Uhlenbeck compactification. In 4 , we discuss the role this localization plays in producing a formula for the error in (1.2).

We do not expect such a formula for the error to be explicit but rather one of the type appearing in the Kotschick-Morgan conjecture ([19]) or in the $\mathrm{SO}(3)$ monopole cobordism formula (8) $)$. The $\mathrm{SO}(3)$-monopole cobordism formula gives relations between both the Donaldson and Seiberg-Witten invariants and between the spin and Seiberg-Witten invariants, but these relations are given by unknown polynomials with universal coefficients depending on topological invariants of $X$. These coefficients have been determined in some cases but not all. Having a direct relation between the Donaldson and spin invariants would give us additional leverage for determining these coefficients. (It is worth noting that since the initial submission of this paper, the results of 9 , 17 have reduced the work of determining these polynomials in the cases necessary to prove Witten's conjecture, [32, to the analysis needed in [8.) It is possible that this additional leverage will reveal new, topological constraints of the type appearing in [6] on these invariants of the smooth structure.

Finally, we note that these computations, in particular Proposition [3.10, have had additional applications in 25].

\section{Preliminaries}

2.1. ASD moduli space. Let $E_{\kappa}^{w} \rightarrow X$ be a complex, rank-two, Hermitian vector bundle over a smooth, closed, oriented four-manifold with $b^{1}(X)=0, w=c_{1}\left(E_{\kappa}^{w}\right)$ 
and $\kappa=\left\langle c_{2}\left(E_{\kappa}^{w}\right)-\frac{1}{4} c_{1}\left(E_{\kappa}^{w}\right)^{2},[X]\right\rangle$. Fix a connection $A^{w}$ on the line bundle $\operatorname{det}\left(E_{\kappa}^{w}\right)$. For $k \geq 2$, define $\mathcal{A}_{\kappa}^{w}$ to be the $L_{k}^{2}$ completion of the space of unitary connections $A$ on $E_{\kappa}^{w}$ with $A^{\text {det }}=A^{w}$. Let $\mathcal{A}_{\kappa}^{w, *} \subset \mathcal{A}_{\kappa}^{w}$ be the irreducible connections. Define $\mathcal{G}_{\kappa}^{w}$ to be the $L_{k+1}^{2}$ completion of the group of special-unitary gauge transformations of $E_{\kappa}^{w}$. Define quotient spaces,

$$
\mathcal{B}_{\kappa}^{w}=\mathcal{A}_{\kappa}^{w} / \mathcal{G}_{\kappa}^{w}, \quad \mathcal{B}_{\kappa}^{w, *}=\mathcal{A}_{\kappa}^{w, *} / \mathcal{G}_{\kappa}^{w} .
$$

For $A \in A_{\kappa}^{w}$, let $F_{A}$ be the curvature of $A$, let $\left(F_{A}^{+}\right)_{0}$ be the self-dual and trace-free component of the curvature determined by a Riemannian metric $g$ on $X$,

$$
\left(F_{A}^{+}\right)_{0} \in \Omega^{+}(\mathfrak{s u}(E)) .
$$

Then define the moduli space of projectively anti-self-dual connections on $E_{\kappa}^{w}$ by

$$
M_{\kappa}^{w}=\tilde{M}_{\kappa}^{w} / \mathcal{G}_{\kappa}^{w}, \quad \text { where } \quad \tilde{M}_{\kappa}^{w}=\left\{A \in \mathcal{A}_{\kappa}^{w}:\left(F_{A}^{+}\right)_{0}=0\right\} .
$$

The following result is well known.

Theorem 2.1 ([15, 4]). For generic choices of a metric $g$ on $X$, the moduli space $M_{\kappa}^{w}$ is a smooth, orientable manifold of dimension

$$
d(\kappa)=8 \kappa-\frac{3}{2}(\chi(X)+\sigma(X))
$$

where $\chi(X)$ is the Euler characteristic and $\sigma(X)$ the signature of $X$.

2.2. $\operatorname{Spin}^{u}$ structures and Dirac operators. As defined in [27, 20, 11, let $\left(W, \rho_{W}\right)$ be a $\operatorname{spin}^{c}$ structure on a four-dimensional, Riemannian manifold $(X, g)$. Let $E \rightarrow X$ be a rank-two, complex vector bundle. A spin ${ }^{u}$ structure on $X$ is the pair $\mathfrak{t}=(V, \rho)$, where $V=W \otimes E$ and $\rho: T^{*} X \rightarrow \operatorname{Hom}(V)$ is defined by $\rho=\rho_{W} \otimes \operatorname{id}_{E}$. We define additional bundles $V^{ \pm}=W^{ \pm} \otimes E$ and $\mathfrak{g}_{\mathfrak{t}}=\mathfrak{s u}(E)$. A more intrinsic definition of these concepts appears in [12, §2.1.3]. We define characteristic classes of $\mathfrak{t}$ by

$$
p_{1}(\mathfrak{t})=p_{1}\left(\mathfrak{g}_{\mathfrak{t}}\right), \quad c_{1}(\mathfrak{t})=c_{1}(E)+c_{1}\left(W^{+}\right), \quad w_{2}(\mathfrak{t})=w_{2}\left(\mathfrak{g}_{\mathfrak{t}}\right) .
$$

A spin connection $A_{W}$ on $W$ determines a bijection, $A \mapsto A \otimes A_{W}$, between $\mathcal{A}_{\kappa}^{w}$ and connections on $V$ respecting the Clifford multiplication map (see [11, §2.1.4]). This map associates a Dirac operator $D_{A}=D_{A \otimes A_{W}}: L_{k}^{2}\left(V^{+}\right) \rightarrow L_{k-1}^{2}\left(V^{-}\right)$to every $A \in \mathcal{A}_{\kappa}^{w}$. The index of $D_{A}$ is ([10, Prop. 2.28])

$$
n_{a}(\mathfrak{t})=\frac{1}{4}\left(p_{1}(\mathfrak{t})+c_{1}(\mathfrak{t})^{2}-\sigma(X)\right) .
$$

2.3. The degeneracy locus. For $\mathfrak{t}$ a $\operatorname{spin}^{u}$ structure on $X$ with characteristic classes $\kappa=-\frac{1}{4} p_{1}(\mathfrak{t}), \Lambda=c_{1}(\mathfrak{t})$, and $w$ an integer lift of $w_{2}(\mathfrak{t})$, define the degeneracy locus

$$
J_{\kappa}^{\Lambda, w}=\left\{[A] \in M_{\kappa}^{w}: \operatorname{dim} \operatorname{Ker} D_{A}=1\right\} .
$$

Theorem 2.2 ([5, [31]). Let $\mathfrak{t}$ be a spin ${ }^{u}$ structure on $X$ with $n_{a}(\mathfrak{t}) \leq 0, \Lambda=$ $c_{1}(\mathfrak{t}), \kappa=-\frac{1}{4} p_{1}(\mathfrak{t})$, and let $w$ be an integer lift of $w_{2}(\mathfrak{t})$. For generic choices of perturbations, $J_{\kappa}^{\Lambda, w}$ is a smooth submanifold of $M_{\kappa}^{w}$ of real codimension $2\left(1-n_{a}(\mathfrak{t})\right)$. The fiber of the normal bundle of $J_{\kappa}^{\Lambda, w}$ in $M_{\kappa}^{w}$ at $[A] \in J_{\kappa}^{\Lambda, w}$ is given by

$$
\mathcal{N}_{A}=\operatorname{Hom}_{\mathbb{C}}\left(\operatorname{Ker} D_{A}, \operatorname{Coker} D_{A}\right) \text {. }
$$

Hence,

$$
\operatorname{dim} J_{\kappa}^{\Lambda, w}=d_{a}(\mathfrak{t})+2 n_{a}(\mathfrak{t})-2
$$


Definition 2.3. Let $\mathcal{N}_{\kappa}^{\Lambda, w} \rightarrow J_{\kappa}^{\Lambda, w}$ be the complex rank $1-n_{a}(\mathfrak{t})$ normal bundle of $J_{\kappa}^{\Lambda, w}$ in $M_{\kappa}^{w}$ with fiber over $[A] \in J_{\kappa}^{\Lambda, w}$ given by $\mathcal{N}_{A}$ as defined in (2.4).

2.4. The Koschorke-Porteous formula for families of Fredholm operators. Let $\mathcal{F}_{n}\left(H, H^{\prime}\right)$ be the space of complex linear, index $n$ Fredholm operators $L$ : $H \rightarrow H^{\prime}$, where $H$ and $H^{\prime}$ are Banach spaces. Let $\mathcal{F}_{k, n}\left(H, H^{\prime}\right) \subset \mathcal{F}_{n}\left(H, H^{\prime}\right)$ be the subspace of operators with a $k$-dimensional kernel. In [18, §3.1], Koschorke constructs a cohomology class $\chi_{k, k-n} \in H^{2 k(k-n)}\left(\mathcal{F}_{n}\left(H, H^{\prime}\right)\right)$ given, essentially, as the Thom class of the normal bundle of $\mathcal{F}_{k, n}\left(H, H^{\prime}\right)$ in $\mathcal{F}_{n}\left(H, H^{\prime}\right)$. Any family, $B$, of complex linear, index $n$ Fredholm operators between separable Hilbert spaces defines a homotopy class of maps $f: B \rightarrow \mathcal{F}_{n}\left(H, H^{\prime}\right)$ and characteristic classes, $f^{*} \chi_{k, k-n}$. If the family is compact with index bundle $V \in K(B)$, the characteristic classes $f^{*} \chi_{k, k-n}$ are related to the Chern classes of $V$ by the equality (see [18, Thm. $5.2])$

$$
f^{*} \chi_{1-n, 1}=(-1)^{1-n} c_{1-n}(V) .
$$

Further discussions and applications of (2.6) appear in [1, p. 110].

\section{Chern Classes of the index Bundle}

To apply (2.6) to prove Theorem 1.1 we use the following version of the AtiyahSinger index theorem for families.

Theorem 3.1 ([2], 4, Thm. 5.1.16]). Let $\mathcal{E} \rightarrow X \times B$ be a family of vector bundles with connections over the four-manifold $X$ parameterized by $B$. Let $(W, \rho)$ be a spin $^{c}$ structure on $X$. Let $\mathbb{D}$ be the index bundle of the family of Dirac operators,

$$
D_{b}: \Omega^{0}\left(\left.W^{-} \otimes \mathcal{E}\right|_{X \times b}\right) \rightarrow \Omega^{0}\left(\left.W^{+} \otimes \mathcal{E}\right|_{X \times b}\right),
$$

as $b$ varies in $B$. Then,

$$
\operatorname{ch}(\mathbb{D})=-e^{c_{1}\left(W^{+}\right) / 2} \operatorname{ch}(\mathcal{E})\left(1-\frac{1}{24} p_{1}(X)\right) /[X] .
$$

The first obstruction to applying Theorem 3.1 to the family of Dirac operators $D_{A}$ for $[A] \in M_{\kappa}^{w}$ is that the bundle playing the role of $\mathcal{E}$,

$$
\mathbb{E}_{\kappa}^{w}=\mathcal{A}_{\kappa}^{w, *} \times \mathcal{G}_{\kappa}^{w} E_{\kappa}^{w} \rightarrow \mathcal{B}_{\kappa}^{w, *} \times X
$$

is not a vector bundle because for $A \in \mathcal{A}_{\kappa}^{w, *}, \operatorname{Stab}_{A}=\{ \pm 1\} \leq \mathcal{G}_{\kappa}^{w}$. As discussed in [23. pp. 34-35], the approach to this problem in 23 only works when the universal $\mathrm{SO}(3)$ bundle defined in (3.3) admits a $\mathrm{U}(2)$ lifting. To overcome this difficulty in general, we introduce a larger space with the same rational homotopy type.

Lemma 3.2. Let $\mathbf{S}$ be the unit sphere in $L^{2}\left(V^{+}\right)$. Then, the map

$$
\pi_{S}: \mathcal{B}_{\kappa}^{w, *}(S)=\mathcal{A}_{\kappa}^{w, *} \times \mathcal{G}_{\kappa}^{w} \mathbf{S} \rightarrow \mathcal{B}_{\kappa}^{w, *}
$$

is a $K\left(\mathbb{Z}_{2}, 1\right)$-bundle and hence defines an isomorphism in rational homotopy.

We define the following subspaces of $\mathcal{B}_{\kappa}^{w, *}(S)$ analogously:

$$
M_{\kappa}^{w}(S)=\pi_{S}^{-1}\left(M_{\kappa}^{w}\right), \quad J_{\kappa}^{\Lambda, w}(S)=\pi_{S}^{-1}\left(J_{\kappa}^{\Lambda, w}\right) .
$$

The following is immediate from Theorem 2.2 and the surjectivity of $\pi_{S}$.

Lemma 3.3. The space $J_{\kappa}^{\Lambda, w}(S)$ is a smooth submanifold of $M_{\kappa}^{w}(S)$ with normal bundle given by $\pi_{S}^{*} \mathcal{N}_{\kappa}^{\Lambda, w}$. 
For $K_{\kappa}^{w} \subset M_{\kappa}^{w}$ a compact subset, consider the family of Fredholm operators parameterized by $(A, \Phi) \in \mathcal{A}_{\kappa}^{w} \times \mathbf{S}$ with $[A] \in K_{\kappa}^{w}$ :

$$
(A, \Phi) \mapsto D_{A}^{*}: L_{k}^{2}\left(V^{-}\right) \rightarrow L_{k-1}^{2}\left(V^{+}\right) .
$$

This is not a compact family, but it admits a stabilization in the sense that there is a surjective map from a finite-dimensional, trivial bundle onto the cokernels of these operators. The construction of the stabilization follows immediately from the independence of $D_{A}$ from $\Phi$ and the gauge equivariance of the Dirac operator (see [11, Thm. 3.19] for an example of this type of argument). This stabilization allows the following definition of an index bundle for this family of operators.

Definition 3.4. For $K_{\kappa}^{w} \subset M_{\kappa}^{w}$ a compact subset, let $\mathbb{D}_{\kappa}^{\Lambda, w} \in K\left(\pi_{S}^{-1}\left(K_{\kappa}^{w}\right)\right)$ be the index bundle of the family of operators defined by (3.1).

Then Lemma 3.3 and (2.6) give us:

Lemma 3.5. Let $K_{\kappa}^{w} \subset M_{\kappa}^{w}$ be a compact subset. Let $\mathfrak{t}$ be a spin ${ }^{u}$ structure with $n_{a}(\mathfrak{t}) \leq 0$, where $n_{a}(\mathfrak{t})$ is the Dirac operator index computed in (2.2). Then,

$$
\begin{aligned}
\pi_{S}^{*} c_{1-n_{a}(\mathfrak{t})}\left(\left.N_{\kappa}^{\Lambda, w}\right|_{K_{\kappa}^{w}}\right) & =\left.c_{1-n_{a}(\mathfrak{t})}\left(\pi_{S}^{*} \mathcal{N}_{\kappa}^{\Lambda, w}\right)\right|_{\pi_{S}^{-1}\left(K_{\kappa}^{w}\right)} \\
& =(-1)^{1-n_{a}(\mathfrak{t})} c_{1-n_{a}(\mathfrak{t})}\left(\mathbb{D}_{\kappa}^{\Lambda, w}\right) .
\end{aligned}
$$

To apply Theorem 3.1 to compute $\operatorname{ch}\left(\mathbb{D}_{\kappa}^{\Lambda, w}\right)$, we observe that the index bundle $\mathbb{D}_{\kappa}^{\Lambda, w}$ is defined as the index bundle of a family of Dirac operators obtained by twisting the $\operatorname{spin}^{c}$ structure $(W, \rho)$ by

$$
\mathbb{E}_{\kappa}^{w}(S)=\mathcal{A}_{\kappa}^{w} \times \mathbf{S} \times \mathcal{G}_{\kappa}^{w} E_{\kappa}^{w} \rightarrow \mathcal{B}_{\kappa}^{w, *} \times X .
$$

Thus we must compute $\operatorname{ch}\left(\mathbb{E}_{\kappa}^{w}(S)\right)$. To this end, we introduce the following cohomology classes. Recall that for $\beta \in H_{\bullet}(X ; \mathbb{R}), \mu(\beta) \in H^{4-\bullet}\left(\mathcal{B}_{\kappa}^{w, *} ; \mathbb{R}\right)$ was defined in 3 by

$$
\mu(\beta)=-\frac{1}{4} p_{1}\left(\mathbb{F}_{\kappa}^{w}\right) / \beta, \quad \text { where } \quad \mathbb{F}_{\kappa}^{w}=\mathcal{A}_{\kappa}^{w, *} \times \mathcal{G}_{\kappa}^{w} \mathfrak{s u}\left(E_{\kappa}^{w}\right) \rightarrow \mathcal{B}_{\kappa}^{w, *} \times X .
$$

If we define

$$
\mathbb{F}_{\kappa}^{w}(S)=\pi_{S}^{*} \mathbb{F}_{\kappa}^{w}=\mathcal{A}_{\kappa}^{w} \times \mathbf{S} \times \mathcal{G}_{\kappa}^{w} \mathfrak{g}_{\mathfrak{t}},
$$

then $\pi_{S}^{*} \mu(\beta)=-\frac{1}{4} p_{1}\left(\mathbb{F}_{\kappa}^{w}(S)\right) / \beta$.

Lemma 3.6. Assume $X$ is a smooth, closed, oriented four-manifold with $b^{1}(X)=$ 0 . Let $E_{\kappa}^{w} \rightarrow X$ be a complex, rank-two vector bundle with $c_{1}\left(E_{\kappa}^{w}\right)=w$ and $c_{2}\left(E_{\kappa}^{w}\right)=\kappa+\frac{1}{4} w^{2}$. Let $\beta_{1}, \ldots, \beta_{d}$ be a basis for $H_{2}(X) /$ Tor and let $x \in H_{0}(X)$ be a generator. Let $\beta_{i}^{*}=\operatorname{PD}\left[\beta_{i}\right]$. Let $Q_{i j}=Q_{X}\left(\beta_{i}, \beta_{j}\right)$ and let $P^{i j}$ be the inverse matrix of $Q_{i j}$. Let $\mu_{i}=\pi_{S}^{*} \mu\left(\beta_{i}\right)$ and $\wp=\pi_{S}^{*} \mu(x)$. Then, for $\mathbb{E}_{\kappa}^{w}(S)$ and $\mathbb{F}_{\kappa}^{w}(S)$ as defined in (3.2) and (3.4) respectively,

$$
\begin{aligned}
& c_{1}\left(\mathbb{E}_{\kappa}^{w}(S)\right)=1 \times w, \\
& p_{1}\left(\mathbb{F}_{\kappa}^{w}(S)\right)=-4 \wp \times 1-4 \sum_{i, j} P^{i j} \mu_{i} \times \beta_{j}^{*}-4 \kappa(1 \times \mathrm{PD}[x]),
\end{aligned}
$$

as elements of rational cohomology.

Proof. The first equality follows from observing that

$$
\begin{aligned}
\operatorname{det}\left(\mathbb{E}_{\kappa}^{w}(S)\right) & =\mathcal{A}_{\kappa}^{w} \times \mathbf{S} \times{ }_{\left(\mathcal{G}_{\kappa}^{w}, \operatorname{det}\right)} \operatorname{det}(E) \simeq\left(\mathcal{A}_{\kappa}^{w} \times{ }_{\mathcal{G}_{\kappa}^{w}} \mathbf{S}\right) \times \operatorname{det}(E) \\
& =\mathcal{B}_{\kappa}^{w, *}(S) \times \operatorname{det}(E) .
\end{aligned}
$$


The second equality then follows from [23, 5.4.1], which we now review. By Lemma 3.2, $\pi_{S}^{*}$ is an isomorphism in real cohomology, so it suffices to compute $p_{1}\left(\mathbb{F}_{\kappa}^{w}\right)$. By [4, Prop. 5.1.15], $H^{\bullet}\left(\mathcal{B}_{\kappa}^{w, *} ; \mathbb{Q}\right)$ is a polynomial algebra in $\mu\left(\beta_{i}\right)$ and $\mu(x)$, so we can write

$$
p_{1}\left(\mathbb{F}_{\kappa}^{w}\right)=a_{0} \mu(x) \times 1+\sum_{i, j} a_{i, j} \mu\left(\beta_{i}\right) \times \beta_{j}^{*}+b_{0} 1 \times x^{*} .
$$

To compute the coefficient $a_{0}$, observe that

$$
p_{1}\left(\left.\mathbb{F}_{\kappa}^{w}\right|_{\mathcal{B}_{\kappa}^{w, *} \times\{x\}}\right)=-4 \mu(x) .
$$

To compute the coefficient $b_{0}$, observe that for $[A] \in \mathcal{B}_{\kappa}^{w, *}$,

$$
p_{1}(\{[A]\} \times X)=p_{1}\left(\mathfrak{g}_{\mathfrak{t}}\right)=-4 \kappa \mathrm{PD}[x] .
$$

Finally, observe that

$$
\begin{aligned}
\mu\left(\beta_{k}\right) & =-\frac{1}{4} p_{1}\left(\mathbb{F}_{\kappa}^{w}\right) / \beta_{k} \\
& =-\frac{1}{4} \sum_{i, j} a_{i, j} \mu\left(\beta_{i}\right) \times \beta_{j}^{*} / \beta_{k} \\
& =-\frac{1}{4} \sum_{i, j} a_{i, j} Q_{j, k} \mu\left(\beta_{i}\right) .
\end{aligned}
$$

The linear independence of $\mu\left(\beta_{i}\right)$ then implies that $a_{i, j}=-4 P^{i j}$, as required.

Lemma 3.6 and the following will yield the Chern character of $\mathbb{E}_{\kappa}^{w}(S)$.

Lemma 3.7. Let $E \rightarrow Y$ be a rank-two, complex Hermitian vector bundle. Let $c_{1}=c_{1}(E)$ and $p_{1}=p_{1}(\mathfrak{s u}(E))$. Then

$$
\operatorname{ch}(E)=2 e^{c_{1} / 2} \sum_{n=1}^{\infty} \frac{p_{1}^{n}}{4^{n}(2 n) !}
$$

Proof. By the splitting principle, we may assume that $E=L_{1} \oplus L_{2}$, where $L_{i} \rightarrow Y$ is a complex line bundle. Let $x=c_{1}\left(L_{1}\right)$ and $y=c_{1}\left(L_{2}\right)$. Then,

$$
\begin{aligned}
\operatorname{ch}(E) & =e^{x}+e^{y} \\
& =e^{(x+y) / 2}\left(e^{(x-y) / 2}+e^{-(x-y) / 2}\right) \\
& =e^{(x+y) / 2}\left(\sum_{n=0}^{\infty} \frac{(x-y)^{n}}{2^{n} n !}+\sum_{n=0}^{\infty} \frac{(-1)^{n}(x-y)^{n}}{2^{n} n !}\right) \\
& =2 e^{(x+y) / 2}\left(\sum_{n=0}^{\infty} \frac{(x-y)^{2 n}}{2^{2 n}(2 n) !}\right)
\end{aligned}
$$

The lemma then follows from the observation that $x+y=c_{1}(E)$ and $(x-y)^{2}=$ $p_{1}(\mathfrak{s u}(E))$.

Corollary 3.8. Continue the notation of Lemma 3.6. Then,

$$
\operatorname{ch}\left(\mathbb{E}_{\kappa}^{w}(S)\right)=2 e^{w / 2} \sum_{n=0}^{\infty} \frac{(-1)^{n}}{(2 n) !}\left(\wp \times 1+\sum_{i, j} P^{i j} \mu_{i} \times \beta_{j}^{*}+\kappa(1 \times \operatorname{PD}[x])\right)^{n} .
$$

Our computation of $\left.\operatorname{ch}\left(\mathbb{D}_{\kappa}^{\Lambda, w}\right)\right)$ requires the following algebraic result. 
Lemma 3.9. Continue the notation of Lemma 3.6. Let $h \in H^{2}(X ; \mathbb{R})$ satisfy $h=\sum_{k=1}^{d} h_{k} \beta_{k}^{*}$. Then,

$$
\begin{aligned}
(1 \times h) & \smile\left(\sum_{i, j} P^{i j} \mu_{i} \times \beta_{j}^{*}\right)=\sum_{k} h_{k} \mu_{k} \times \mathrm{PD}[x] \\
\left(\sum_{i, j} P^{i j} \mu_{i} \times \beta_{j}^{*}\right) & \smile\left(\sum_{k, \ell} P^{k \ell} \mu_{k} \times \beta_{\ell}^{*}\right)=\sum_{i, j} P^{i j}\left(\mu_{i} \smile \mu_{j}\right) \times \operatorname{PD}[x] .
\end{aligned}
$$

Proof. The first equality follows from

$$
h \smile \beta_{j}^{*}=\sum_{k} h_{k} \beta_{k}^{*} \smile \beta_{j}^{*}=\sum_{k} h_{k} Q_{k j} \mathrm{PD}[x]
$$

and the equality $\sum_{j} P^{i j} Q_{j k}=\delta_{k}^{i}$, where $\delta_{k}^{i}$ is the Kronecker delta. The second equality follows from computing

$$
\beta_{j}^{*} \smile \beta_{\ell}^{*}=Q_{j \ell} \operatorname{PD}[x],
$$

and the definition of $P^{i j}$ as the inverse of the matrix $Q_{j \ell}$.

We now compute $\operatorname{ch}\left(\mathbb{D}_{\kappa}^{\Lambda, w}\right)$.

Proposition 3.10. Continue the notation of Lemma 3.6. For $c_{1}(\mathfrak{t})=\sum_{i} \lambda^{i} \beta_{i}^{*}$ define

$$
\mu(\mathfrak{t})=\sum_{i} \lambda^{i} \mu_{i} \quad \text { and } \quad \Omega=\sum_{i, j} P^{i j} \mu_{i} \smile \mu_{j} .
$$

Then

$$
\operatorname{ch}_{2 k}\left(\mathbb{D}_{\kappa}^{\Lambda, w}\right)=-\frac{(-1)^{k}}{(2 k) !}\left(\left(n_{a}(\mathfrak{t})+\frac{2 k \kappa}{2 k+1}\right) \wp^{k}-\frac{k}{2(2 k+1)} \Omega \smile \wp^{k-1}\right),
$$

$$
\operatorname{ch}_{2 k+1}\left(\mathbb{D}_{\kappa}^{\Lambda, w}\right)=\frac{(-1)^{k}}{2(2 k+1) !} \mu(\mathfrak{t}) \smile \wp^{k} .
$$

Proof. Applying Theorem 3.1, Corollary 3.8 and $c_{1}(\mathfrak{t})=c_{1}\left(W^{+}\right)+c_{1}(E)$ yields

$$
\begin{aligned}
\operatorname{ch}\left(\mathbb{D}_{\kappa}^{\Lambda, w}\right) & \\
= & -\operatorname{ch}\left(\mathbb{E}_{\kappa}^{w}(S)\right) e^{c_{1}\left(W^{+}\right) / 2}\left(1-\frac{1}{24} p_{1}(X)\right) /[X] \\
= & -2 e^{c_{1}(\mathfrak{t}) / 2}\left(1-\frac{1}{24} p_{1}(X)\right) \\
& \smile \sum_{n=0}^{\infty} \frac{(-1)^{n}}{(2 n) !}\left(\wp \times 1+\sum_{i, j} P^{i j} \mu_{i} \times \beta_{j}^{*}+\kappa(1 \times \operatorname{PD}[x])\right)^{n} /[X] .
\end{aligned}
$$

The second equality of Lemma 3.9 implies that

$$
\begin{aligned}
& \left(\sum_{i, j} P^{i j} \mu_{i} \times \beta_{j}^{*}+\kappa(1 \times \operatorname{PD}[x])\right)^{n} \\
& \quad= \begin{cases}\sum_{i, j} P^{i j}\left(\mu_{i} \smile \mu_{j}\right) \times \operatorname{PD}[x] & \text { if } n=2, \\
0 & \text { if } n>2 .\end{cases}
\end{aligned}
$$


Using the preceding, we expand the factor in (3.7):

$$
\begin{aligned}
(\wp & \left.\times 1+\sum_{i, j} P^{i j} \mu_{i} \times \beta_{j}^{*}+\kappa(1 \times \mathrm{PD}[x])\right)^{n} \\
= & \wp^{n} \times 1+n\left(\wp^{n-1} \times 1\right) \smile\left(\sum_{i, j} P^{i j} \mu_{i} \times \beta_{j}^{*}+\kappa(1 \times \mathrm{PD}[x])\right) \\
& +\left(\begin{array}{l}
n \\
2
\end{array}\right)\left(\wp^{n-2} \times 1\right) \smile\left(\sum_{i, j} P^{i j} \mu_{i} \times \beta_{j}^{*}+\kappa(1 \times \mathrm{PD}[x])\right)^{2} \\
= & \wp^{n} \times 1+n \sum_{i, j} P^{i j}\left(\wp^{n-1} \smile \mu_{i}\right) \times \beta_{j}^{*}+n \kappa \wp^{n-1} \times \mathrm{PD}[x] \\
& +\left(\begin{array}{l}
n \\
2
\end{array}\right) \sum_{i, j} P^{i j}\left(\wp^{n-2} \smile \mu_{i} \smile \mu_{j}\right) \times \mathrm{PD}[x] .
\end{aligned}
$$

Substituting (3.8) into (3.7) gives

$$
\begin{aligned}
-\operatorname{ch}\left(\mathbb{D}_{\kappa}^{\Lambda, w}\right) & \\
= & 2\left(1+\frac{1}{2} 1 \times c_{1}(\mathfrak{t})+\frac{1}{8} 1 \times c_{1}(\mathfrak{t})^{2}\right)\left(1-1 \times \frac{1}{24} p_{1}(X)\right) \\
& \smile \sum_{n=0}^{\infty} \frac{(-1)^{n}}{(2 n) !}\left(\wp^{n} \times 1+n \sum_{i, j} P^{i j}\left(\wp^{n-1} \smile \mu_{i}\right) \times \beta_{j}^{*}+n \kappa \wp^{n-1} \times \mathrm{PD}[x]\right. \\
& \left.+\left(\begin{array}{c}
n \\
2
\end{array}\right) \sum_{i, j} P^{i j}\left(\wp^{n-2} \smile \mu_{i} \smile \mu_{j}\right) \times \mathrm{PD}[x]\right) /[X] .
\end{aligned}
$$

Applying the first equality of Lemma 3.9 and the definitions of $\mu(\mathfrak{t})$ and $\Omega$ in (3.5) to the preceding yields

$$
\begin{aligned}
-\operatorname{ch}\left(\mathbb{D}_{\kappa}^{\Lambda, w}\right) \\
=\sum_{n=0}^{\infty} \frac{(-1)^{n}}{(2 n) !} \wp^{n} \frac{1}{4}\left(c_{1}(\mathfrak{t})^{2}-\sigma(X)\right)+\sum_{n=1}^{\infty} \frac{(-1)^{n}}{(2 n) !} 2 n \kappa \wp^{n-1} \\
\quad+\sum_{n=1}^{\infty} \frac{(-1)^{n}}{(2 n) !} n \mu(t) \wp^{n-1}+\sum_{n=2}^{\infty} \frac{(-1)^{n}}{(2 n) !} 2\left(\begin{array}{c}
n \\
2
\end{array}\right) \wp^{n-2} \smile \Omega \\
=\sum_{n=0}^{\infty} \frac{(-1)^{n}}{(2 n) !} \frac{1}{4}\left(c_{1}(\mathfrak{t})^{2}-\sigma(X)\right) \wp^{n}-\sum_{n=0}^{\infty} \frac{(-1)^{n}}{(2 n+2) !} 2(n+1) \kappa \wp^{n} \\
\quad-\sum_{n=0}^{\infty} \frac{(-1)^{n}}{(2 n+2) !}(n+1) \mu(t) \smile \wp^{n}+\sum_{\ell=1}^{\infty} \frac{(-1)^{\ell+1}}{(2 \ell+2) !} 2\left(\begin{array}{c}
\ell+1 \\
2
\end{array}\right) \wp^{\ell-1} \smile \Omega .
\end{aligned}
$$


If we observe that $n_{a}(\mathfrak{t})+\kappa=\left(c_{1}(\mathfrak{t})^{2}-\sigma(X)\right) / 4$, then we can write

$$
\begin{aligned}
\operatorname{ch}\left(\mathbb{D}_{\kappa}^{\Lambda, w}\right)= & -\sum_{k=0}^{\infty} \frac{(-1)^{k}}{(2 k) !}\left(\left(n_{a}(\mathfrak{t})+\kappa-\frac{\kappa}{2 k+1}\right) \wp^{k}-\frac{k}{2(2 k+1)} \Omega \smile \wp^{k-1}\right) \\
& +\sum_{k=0}^{\infty} \frac{(-1)^{k}}{2(2 k+1) !} \mu(t) \smile \wp^{k} .
\end{aligned}
$$

This completes the proof of Proposition 3.10.

Proof of Theorem 1.1. Because $\pi_{S}^{*}$ is an isomorphism on rational cohomology, it suffices to compute the pullback by $\pi_{S}$ of the Chern class. After Proposition 3.10 and Lemma 3.5. the only remaining step in the proof of Theorem 1.1 is to compute the Chern class from the Chern character.

If, for a vector bundle $E$, we define $q_{r}(E)=r ! \operatorname{ch}_{r}(E)$ and

$$
C(E)(t)=\sum_{r \geq 0} c_{r}(E) t^{r}, \quad Q(E)(t)=\sum_{r \geq 1} q_{r}(E) t^{r-1}
$$

then these formal power series are related by [24, Eq. (2.10')]

$$
\frac{d}{d t} C(E)(t)=Q(E)(-t) C(E)(t) \quad \text { or } \quad C(E)(t)=\exp \left(\int Q(E)(-t) d t\right) .
$$

The equalities (3.9) are equivalent to Newton's formula, [26, p. 195] or [24, $\left.\left(2.11^{\prime}\right)\right]$,

$$
c_{n}=-\frac{1}{n} \sum_{i=1}^{n}(-1)^{i} q_{i} c_{n-i}
$$

From (3.6), we have

$$
\begin{aligned}
q_{2 k}\left(\mathbb{D}_{\kappa}^{\Lambda, w}\right) & =(-1)^{k+1}\left(\left(n_{a}(\mathfrak{t})+\frac{2 k \kappa}{2 k+1}\right) \wp^{k}-\frac{k}{2(2 k+1)} \Omega \smile \wp^{k-1}\right) \\
q_{2 k+1}\left(\mathbb{D}_{\kappa}^{\Lambda, w}\right) & =\frac{(-1)^{k}}{2} \mu(\mathfrak{t}) \smile \wp^{k} .
\end{aligned}
$$

Equation (3.11) implies that, abbreviating $\mathbb{D}=\mathbb{D}_{\kappa}^{\Lambda, w}$, the power series $Q(\mathbb{D})(t)$ can be written as

$$
Q(\mathbb{D})(t)=\sum_{r=1}^{\infty} t^{r-1} \sum_{i+2 j+2 k=r} q_{i, 2 j, 2 k} \mu(t)^{i} \smile \Omega^{j} \smile \wp^{k},
$$

where, abbreviating $n_{a}=n_{a}(\mathfrak{t})$,

$$
q_{1,0,2 k}=(-1)^{k} 2^{-1}, \quad q_{0,2,2 k-2}=\frac{(-1)^{k} k}{2(2 k+1)}, \quad q_{0,0,2 k}=(-1)^{k+1}\left(n_{a}+\frac{2 k \kappa}{2 k+1}\right) .
$$

and $q_{i, 2 j, 2 k}=0$ if $i+j>1$. If we write

$$
C(\mathbb{D})(t)=\sum_{r=0}^{\infty}\left(\sum_{i+2 j+2 k=r} f_{i, 2 j, 2 k} \mu(t)^{i} \smile \Omega^{j} \smile \wp^{k}\right) t^{r},
$$


then the expression for $q_{i, 2 j, 2 k}$ in (3.12) and Newton's formula (3.10) imply that the coefficients $f_{i, 2 j, 2 k}$ satisfy the recursion relation:

$$
\begin{aligned}
(i+2 j+2 k) f_{i, 2 j, 2 k}= & \sum_{u=1}^{k}(-1)^{u}\left(n_{a}+\frac{2 u \kappa}{2 u+1}\right) f_{i, 2 j, 2 k-2 u} \\
& -\sum_{u=1}^{k+1}(-1)^{u} \frac{u}{2(2 u+1)} f_{i, 2 j-2,2 k-2 u+2} \\
& +\sum_{u=0}^{k} \frac{(-1)^{u}}{2} f_{i-1,2 j, 2 k-2 u} .
\end{aligned}
$$

To find the generating function $C(\mathbb{D})(t)$, we use (3.9) and define

$$
P(\mathbb{D})(t)=\int Q(\mathbb{D})(-t) d t=\sum_{r=1}^{\infty} \frac{(-1)^{r-1}}{r}\left(\sum_{i+2 j+2 k=r} q_{i, 2 j, 2 k} \mu(t)^{i} \smile \Omega^{j} \smile \wp^{k}\right) t^{r} .
$$

Then, we write $P(\mathbb{D})(t)=P_{1}(t)+P_{2}(t)+P_{3,1}(t)+P_{3,2}(t)$, where

$$
\begin{aligned}
P_{1}(t) & =\sum_{k=0}^{\infty} \frac{(-1)^{k}}{2(2 k+1)}\left(\mu \smile \wp^{k}\right) t^{2 k+1}, \\
P_{2}(t) & =\sum_{k=1}^{\infty} \frac{(-1)^{k+1}}{4(2 k+1)}\left(\Omega \smile \wp^{k-1}\right) t^{2 k}, \\
P_{3,1}(t) & =\sum_{k=1}^{\infty} \frac{(-1)^{k} n_{a}}{2 k} \wp^{k} t^{2 k}, \\
P_{3,2}(t) & =\sum_{k=1}^{\infty} \frac{(-1)^{k} \kappa}{(2 k+1)} \wp^{k} t^{2 k} .
\end{aligned}
$$

If we write

$$
P(\mathbb{D})(t)=\sum_{r=0}^{\infty} t^{r} \sum_{i+2 j+2 k=r} m_{i, 2 j, 2 k} \mu^{i} \smile \Omega^{j} \smile \wp^{k}
$$

and define

$$
J(x, y, z)=\sum_{i+2 j+2 k=r} m_{i, 2 j, 2 k} x^{i} y^{2 j} z^{2 k},
$$

then $J=\frac{1}{2} x J_{1}(z)+\frac{1}{4} y^{2} J_{2}(z)+J_{3,1}(z)+J_{3,2}(z)$, where

$$
\begin{aligned}
J_{1}(z) & =\sum_{k=0}^{\infty} \frac{(-1)^{k}}{2 k+1} z^{2 k}=\frac{\tan ^{-1}(z)}{z}, \\
J_{2}(z) & =\sum_{k=1}^{\infty} \frac{(-1)^{k+1}}{2 k+1} z^{2 k-2}=\frac{1}{z^{3}}\left(-\tan ^{-1}(z)+z\right), \\
J_{3,1}(z) & =n_{a} \sum_{k=1}^{\infty} \frac{(-1)^{k}}{2 k} z^{2 k}=-\frac{n_{a}}{2} \ln \left(1+z^{2}\right), \\
J_{3,2}(z) & =\kappa \sum_{k=1}^{\infty} \frac{(-1)^{k}}{2 k+1} z^{2 k}=\kappa\left(\frac{\tan ^{-1}(z)}{z}-1\right) .
\end{aligned}
$$


The preceding and the equality $C(\mathbb{D})(t)=\exp (P(\mathbb{D})(t))$ from $(3.9)$ then complete the proof of Theorem 1.1.

\section{Comparing the Spin AND Donaldson invariants}

We now discuss the sources of the error in the equality (1.2) and the role of Theorem 1.1 in deriving a correct formula for the relation between the spin and Donaldson invariants.

Define

$$
\mathbb{A}(X)=\operatorname{Sym}\left(H_{2}(X ; \mathbb{R}) \oplus H_{0}(X ; \mathbb{R})\right) .
$$

Then the $\mu$-map of (3.3) extends to

$$
\mu: \mathbb{A}(X) \rightarrow H^{\bullet}\left(\mathcal{B}_{\kappa}^{w, *} ; \mathbb{R}\right), \quad \mu\left(h^{\delta-2 m} x^{m}\right)=\mu(h)^{\delta-2 m} \smile \mu(x)^{m},
$$

where $h \in H_{2}(X ; \mathbb{R})$ and $x \in H_{0}(X ; \mathbb{Z})$ is a generator. For $z \in \mathbb{A}(X)$, the definition of a geometric representative, in the sense of [21, $\S 2$.(ii)], $\mathcal{V}(z)$, for $\mu(z)$ appears in [21, §2.(ii)]. If we write $\bar{M}_{\kappa}^{w}$ for the Uhlenbeck compactification of $M_{\kappa}^{w}$ and $\overline{\mathcal{V}}(z)$ for the closure of $\mathcal{V}(z)$ in this compactification, then the Donaldson invariant is defined by

$$
D_{X}^{w}\left(h^{\delta-2 m} x^{m}\right)=\#\left(\overline{\mathcal{V}}\left(h^{\delta-2 m} x^{m}\right) \cap \bar{M}_{\kappa}^{w}\right),
$$

where $\delta=4 \kappa-(3 / 4)(\chi+\sigma)$ if $\delta \equiv-w^{2}-3(\chi+\sigma)(\bmod 4)$ and is defined to vanish otherwise.

The error in the equality

$$
E_{X}^{\Lambda, w}(z)=D_{X}^{w}\left(z T_{\kappa}^{\Lambda, w}\right)-P_{X}^{\Lambda, w}(z)
$$

arises from the difference in the geometric representatives $\mathcal{V}\left(T_{\kappa}^{\Lambda, w}\right)$ and $J_{\kappa}^{\Lambda, w}$ for the cohomology class $\mu\left(T_{\kappa}^{\Lambda, w}\right)$ defined following Theorem 1.1. The intersections

$$
\mathcal{V}\left(T_{\kappa}^{\Lambda, w}\right) \cap \mathcal{V}(z) \cap M_{\kappa}^{w} \quad \text { and } \quad J_{\kappa}^{\Lambda, w} \cap \mathcal{V}(z) \cap M_{\kappa}^{w},
$$

which define $D_{X}^{w}\left(T_{\kappa}^{\Lambda, w} z\right)$ and $P_{X}^{\Lambda, w}(z)$ respectively, both have compact support in the interior of a compact subset $N_{\kappa}^{w}(z) \subset \mathcal{V}(z) \cap M_{\kappa}^{w}$. While there is a cobordism between the geometric representatives $\mathcal{V}\left(T_{\kappa}^{\Lambda, w}\right)$ and $J_{\kappa}^{\Lambda, w}$, this cobordism need not be supported in $N_{\kappa}^{w}(z)$. To understand the error (4.1), one must therefore study the ends of $\mathcal{V}(z) \cap M_{\kappa}^{w}$.

The lower strata of $\bar{M}_{\kappa}^{w}$ have the form $M_{\kappa-\ell}^{w} \times \Sigma$, where $\Sigma \subset \operatorname{Sym}^{\ell}(X)$ is a smooth stratum. Because $\overline{\mathcal{V}}(z)$ is, roughly, transverse to the lower strata, the gluing maps of Taubes, [30] (see also [7] or [16, §III.3.4]), present the ends of $\mathcal{V}(z) \cap M_{\kappa}^{w}$ as a fiber bundle,

$$
M \rightarrow \mathcal{V}(z) \cap M_{\kappa}^{w} \rightarrow \overline{\mathcal{V}}(z) \cap\left(M_{\kappa-\ell}^{w} \times \Sigma\right),
$$

where the fiber $M$ is a cone, given by the product of moduli spaces of framed, centered, anti-self-dual connections on $S^{4}$.

In [23, Leung computes the error (4.1) assuming that $n_{a}(\mathfrak{t})=-1$. He constructs an Ozsvathian "cap" (in the sense of [28]) $C_{\kappa}^{\Lambda, w} \subset \mathcal{B}_{\kappa}^{w}$, , for the end of $\mathcal{V}(z) \cap M_{\kappa}^{w}$, using the description in (4.2). If $W_{\kappa}^{\Lambda, w}$ is the intersection of $\mathcal{V}(z) \cap M_{\kappa}^{w}$ with the end (4.2), then

$$
\widehat{\mathcal{V}}=\left(\mathcal{V}(z) \cap M_{\kappa}^{w}-W_{\kappa}^{\Lambda, w}\right) \cup C_{\kappa}^{\Lambda, w}
$$

will be a compact subspace of $\mathcal{B}_{\kappa}^{w, *}$ giving the equality

$$
\#\left(J_{\kappa}^{\Lambda, w} \cap \widehat{\mathcal{V}}\right)=\#\left(\mathcal{V}\left(T_{\kappa}^{\Lambda, w}\right) \cap \widehat{\mathcal{V}}\right) .
$$


Leung then argues that $\mathcal{V}\left(T_{\kappa}^{\Lambda, w}\right) \cap C_{\kappa}^{\Lambda, w}$ is empty, so the right-hand side of (4.3) is given by $D_{X}^{w}\left(z T_{\kappa}^{\Lambda, w}\right)$ while the left-hand side of (4.3) is given by

$$
P_{\kappa}^{\Lambda, w}(z)+\#\left(J_{\kappa}^{\Lambda, w} \cap C_{\kappa}^{\Lambda, w}\right) .
$$

An excision argument (see [4, Prop. 7.1.32] and [23, $§ 4.3 \& \S 6.3]$ ) shows that the intersection number

$$
\#\left(J_{\kappa}^{\Lambda, w} \cap C_{\kappa}^{\Lambda, w}\right)
$$

is independent of the manifold $X$ and can be computed using examples where both sides of the equality (4.1) are known. This argument yields

Theorem 4.1 ([23]). Continue the hypotheses and notation of Theorem [1.1. If $n_{a}(\mathfrak{t})=-1$, then

$$
\begin{aligned}
& D_{X}^{w}\left(h^{\delta-2 m} x^{m} T_{\kappa}^{\Lambda, w}\right)-P_{X}^{w, \Lambda}\left(h^{\delta-2 m} x^{m}\right) \\
& \quad=\frac{1}{12}\left(\begin{array}{c}
\delta-2 m \\
2
\end{array}\right) D_{X}^{w}\left(h^{\delta-2 m} x^{m}\right) Q_{X}(h)+\frac{1}{12} m D_{X}^{w}\left(h^{\delta-2 m} x^{m-1}\right) .
\end{aligned}
$$

Some partial computations for the case $n_{a}(\mathfrak{t})=-2$ also appear in [23]. However, computing the error (4.1) for general $n_{a}(\mathfrak{t})$ presents some technical challenges. For $n_{a}(\mathfrak{t})<-2$, the closure $\overline{\mathcal{V}}(z) \cap \bar{M}_{\kappa}^{w}$ intersects more than one lower stratum of $\bar{M}_{\kappa}^{w}$, and thus more than one open set of the form (4.2) is needed to cover the ends of $\mathcal{V}(z) \cap M_{\kappa}^{w}$. Constructing caps in the general case is thus far more challenging, not only because of the greater topological complexity of the picture (4.2) for lower strata but also because the open sets covering the ends overlap. Some approaches to this type of problem involving multiple open sets have appeared in [19, 14, 8, 22.

Thus, while Theorem 1.1 does not give a general relation between the Donaldson and spin invariants, this result does allow one to localize the error (4.1) near the lower strata of $\bar{M}_{\kappa}^{w}$. The examples computed by Leung in [23] and similar computations for the Kotschick-Morgan conjecture ([22]) and for the $\mathrm{SO}(3)$ monopole cobordism (13]) suggest that the error (4.1) has the form described in the following conjecture.

Conjecture 4.2. Continue the notation and hypotheses of Theorem 1.1, The error of (4.1) is given by

$$
\begin{aligned}
E_{X}^{\Lambda, w}\left(h^{\delta-2 m} x^{m}\right) & \\
= & \sum_{\ell=0}^{\left[\left(1-n_{a}\right) / 2\right]} \sum_{i+2 j+2 k \leq 2 \ell} D_{X}^{w}\left(h^{\delta-2 m-i-2 j} x^{m-k} \mathcal{O}_{i, j, k, \ell}^{\delta, \Lambda, w}\right) Q_{X}(h)^{j}\langle\Lambda, h\rangle^{i},
\end{aligned}
$$

where $\mathcal{O}_{i, j, k, \ell}^{\delta, \Lambda, w}$ is an expression in the characteristic classes of $\mathbb{D}_{\kappa-\ell}^{\Lambda, w}$ depending only on the homotopy type of $X$ and the indices $\delta, i, j, k, \ell$. If $1-n_{a}=2 p$, then the term with the highest power of $Q_{X}$ in (4.5) is given by

$$
\frac{1}{12} \frac{(\delta-2 m) !}{(\delta-2 m-2 p) ! p ! 2^{p}} D_{X}^{w}\left(h^{\delta-2 m-2 p} x^{m}\right) Q_{X}(h)^{p} .
$$

\section{REFERENCES}

1. M. F. Atiyah and J. D. S. Jones, Topological aspects of Yang-Mills theory, Comm. Math. Phys. 61 (1978), 97-118. MR503187 (80j:58021)

2. M. F. Atiyah and I. M. Singer, Index of elliptic operators. IV, Ann. Math. 93 (1971), 119-138. MR.0279833(43:5554) 
3. S. K. Donaldson, Polynomial invariants for smooth four-manifolds, Topology 29 (1990), 257315. MR 1066174 (92a:57035)

4. S. K. Donaldson and P. B. Kronheimer, The geometry of four-manifolds, Oxford Univ. Press, Oxford, 1990. MR 1079726(92a:57036)

5. P. M. N. Feehan, Generic metrics, irreducible rank-one PU(2) monopoles, and transversality, Comm. Anal. Geom. 8 (2000), 905-967. MR1846123 (2002m:57041)

6. P. M. N. Feehan, P. B. Kronheimer, T. G. Leness, and T. S. Mrowka, PU(2) monopoles and a conjecture of Mariño, Moore, and Peradze, Math. Res. Lett. 6 (1999), 169-182. MR.1689207 (2000f:57035)

7. P. M. N. Feehan and T. G. Leness, Donaldson invariants and wall-crossing formulas. I: Continuity of gluing and obstruction maps, submitted to a print journal, math.DG/9812060 (v3).

8. _ An SO(3)-monopole cobordism formula relating Donaldson and Seiberg-Witten in-

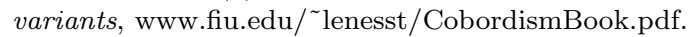

9. P. M. N. Feehan and T. G. Leness, Witten's conjecture for many four-manifolds of simple type, arXiv:math/0609530.

10. _ $P U(2)$ monopoles. I: Regularity, Uhlenbeck compactness, and transversality, J. Differential Geom. 49 (1998), 265-410. MR1664908 (2000e:57052)

11. _ $\mathrm{PU}(2)$ monopoles and links of top-level Seiberg-Witten moduli spaces, J. Reine Angew. Math. 538 (2001), 57-133. MR 1855754 (2002f:57067)

12., $\mathrm{PU}(2)$ monopoles. II. Top-level Seiberg-Witten moduli spaces and Witten's conjecture in low degrees, J. Reine Angew. Math. 538 (2001), 135-212. MR 1855755 (2002f:57068)

13. , $\mathrm{SO}(3)$ monopoles, level-one Seiberg-Witten moduli spaces, and Witten's conjecture in low degrees, Proceedings of the 1999 Georgia Topology Conference (Athens, GA), vol. 124, 2002, pp. 221-326. MR1936209 (2003i:57049)

14. _ $\mathrm{SO}(3)$-monopoles: The overlap problem, Geometry and topology of manifolds, Fields Inst. Commun., vol. 47, Amer. Math. Soc., Providence, RI, 2005, pp. 97-118. MR.2189928 (2006g:57058)

15. D. Freed and K. K. Uhlenbeck, Instantons and four-manifolds, 2nd ed., Springer, New York, 1991. MR1081321 (91i:57019)

16. R. Friedman and J. W. Morgan, Smooth four-manifolds and complex surfaces, Springer, Berlin, 1994. MR1288304 (95m:57046)

17. Lothar Göttsche, Hiraku Nakajima, and Kōta Yoshioka, Donaldson = Seiberg-Witten from Mochizuki's formula and instanton counting, Publ. Res. Inst. Math. Sci. 47 (2011), no. 1, 307-359. MR2827729 (2012f:14085)

18. U. Koschorke, Infinite-dimensional K-theory and characteristic classes of Fredholm bundle maps, Global Analysis (F. E. Browder, ed.), Proc. Symp. Pure Math., vol. 15, Amer. Math. Soc., Providence, RI, 1970, pp. 95-133. MR0279838 (43:5559)

19. D. Kotschick and J. W. Morgan, SO(3) invariants for four-manifolds with $b^{+}=1$. II, J. Differential Geom. 39 (1994), 433-456. MR1267898 (95g:57047)

20. P. B. Kronheimer and T. Mrowka, Monopoles and three-manifolds, New Mathematical Monographs, Cambridge University Press, Cambridge, 2007. MR.2388043 (2009f:57049)

21. P. B. Kronheimer and T. S. Mrowka, Embedded surfaces and the structure of Donaldson's polynomial invariants, J. Differential Geom. 43 (1995), 573-734. MR1338483 (96e:57019)

22. T. G. Leness, Donaldson wall-crossing formulas via topology, Forum Math. 11 (1999), 417457. MR 1699168 (2000g:57044)

23. W.-M. R. Leung, On spin ${ }^{c}$ invariants of four-manifolds, Ph.D. thesis, Oxford University, 1995.

24. I. G. Macdonald, Symmetric functions and Hall polynomials, second ed., Oxford Mathematical Monographs, Oxford Science Publications, The Clarendon Press, Oxford University Press, New York, 1995, With contributions by A. Zelevinsky. MR.1354144 (96h:05207)

25. A. Malmendier and K. Ono, SO(3)-Donaldson invariants of $\overline{C P}^{2}$ and mock theta functions, arXiv:08.08.1442.

26. J. W. Milnor and J. D. Stasheff, Characteristic classes, Princeton Univ. Press, Princeton, NJ, 1974. MR0440554(55:13428)

27. J. W. Morgan, The Seiberg-Witten equations and applications to the topology of smooth fourmanifolds, Princeton Univ. Press, Princeton, NJ, 1996. MR1367507 (97d:57042) 
28. P. S. Ozsváth, Some blowup formulas for SU(2) Donaldson polynomials, J. Differential Geom. 40 (1994), 411-447. MR.1293659 (95e:57054)

29. V. Y. Pidstrigatch and A. N. Tyurin, Invariants of the smooth structure of an algebraic surface arising from the Dirac operator, Russian Acad. Sci. Izv. Math. 40 (1993), 267-351. MR.1180377 (93m:14036)

30. C. H. Taubes, Self-dual connections on 4-manifolds with indefinite intersection matrix, J. Differential Geom. 19 (1984), 517-560. MR755237(86b:53025)

31. A. Teleman, Moduli spaces of PU(2)-monopoles, Asian J. Math. 4 (2000), 391-435. MR1797591 (2001m:57059)

32. E. Witten, Monopoles and four-manifolds, Math. Res. Lett. 1 (1994), 769-796. MR.1306021 (96d:57035)

Department of Mathematics, Florida International University, Miami, Florida 33199 E-mail address: lenesst@fiu.edu

$U R L:$ http://www.fiu.edu/ lenesst 